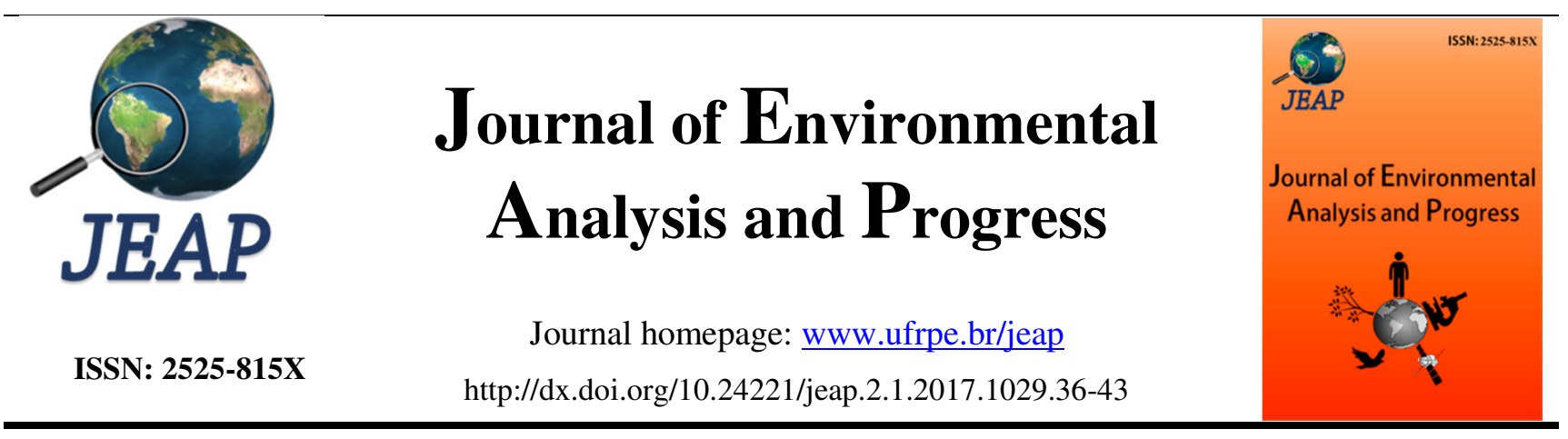

\title{
Influência do uso e ocupação do solo na qualidade da água de nascentes - Macroinvertebrados bentônicos como bioindicadores
}

\section{Influence of land use and occupation in the quality of water sources - Macroinvertebrates benthic as bioindicators}

\author{
Marilene Oliveira dos Santos ${ }^{\mathrm{a}}$, Sandra Maria de Melo ${ }^{\mathrm{b}}$ \\ ${ }^{a}$ Universidade Paulista-UNIP, Av. Baguaçu, n. 1939, Jardim Alvorada, Araçatuba, São Paulo, Brasil. CEP: 16018-555. \\ E-mail: marilene.bio.ata@gmail.com. \\ b Universidade Estadual de Maringá-UEM, Av. Colombo, n. 5790, Paraná, Brasil. CEP: 87020-900. E-mail: \\ sandrammelo@yahoo.com.br.
}

A R T I C L E I N F O

Recebido 01 Nov 2016

Aceito 26 Dez 2016

Publicado 31 Jan 2017

\begin{abstract}
A B S T R A C T
Currently aquatic ecosystems have been altered significantly making it necessary the use of biological monitoring for these environments. In this perspective, this study aimed to analyze the influence of the use and occupation in sub-basin water quality of the Melo Machado Stream, in Araçatuba/SP, using benthic macroinvertebrates as bioindicators. The methodology was developed by means of satellite images geoprocessing, visit in locus and laboratory analysis. The results obtained in this study showed a decrease in the diversity of individuals directly proportional to the degree of land use and occupation, highlighting its impact on water quality in each sampling station. It is noteworthy that the results obtained in this study may support actions and strategies for environmental management of aquatic environments, ensuring the recovery of these environments are important elements in environmental quality.
\end{abstract}

Keywords: Aquatic ecosystems, bioindicators, macroinvertebrates benthic.

\section{R E S U M O}

Atualmente os ecossistemas aquáticos têm sido alterados de maneira significativa tornando-se necessário o uso de medidas biológicas para monitoramento desses ambientes. Nesta perspectiva, o presente estudo objetivou analisar a influência do uso e ocupaçãodo solo na qualidade da água da sub-bacia do Córrego Machado de Melo, no município de Araçatuba/SP, utilizandomacroinvertebrados bentônicos como bioindicadores. A metodologia foi desenvolvida por meio de geoprocessamento de imagens desatélite, visita in locus e análises laboratoriais. Os resultados obtidos no presenteestudo revelaram uma diminuição da diversidade de indivíduos diretamenteproporcional ao grau do uso e ocupação do solo, evidenciando o seu impacto naqualidade da água em cada estação amostral. Vale ressaltar que os resultados obtidos nesta pesquisapoderão subsidiar ações e estratégias para a gestão ambiental de ambientesaquáticos continentais, assegurando a recuperação desses ambientes queconstituem importantes elementos na qualidade ambiental.

Palavras-Chave: Ecossistemas aquáticos, bioindicadores, macroinvertebrados bentônicos.

\section{Introdução}

A qualidade das águas de uma bacia hidrográfica pode ser influenciada por diversos fatores, dentre os impactos ambientais que podem alterar o ecossistema aquáticos. Rebouças et al. (2002) relatam que o desmatamento, o uso do solo 
para monoculturas, a descarga de efluentes urbanos e a construção de barragens destacam-se em escala local e regional.

Estudos que visam analisar a relação entre a mudança do uso do solo e a qualidade da água utilizando técnicas de Geoprocessamento de Imagens de Satélite (SIG) observam uma piora na qualidade de água com o aumento do uso e ocupação do solo. As classes de uso e ocupação mais utilizadas são: Pastagem (P), Lavouras Temporárias (LT), Florestal (F) e Área urbana (AU) (Ometo et al., 2000).

Em bacias hidrográficas, o monitoramento é de suma importância e procura caracterizar aspectos relevantes que permitam diagnosticar as mudanças que ocorrem no uso e ocupação do solo, tornando possível avaliar os efeitos das atividades humanas sobre esses ecossistemas (Queiroz et al., 2010). Para que haja um manejo integrado, o monitoramento ambiental deve incluir, além dos parâmetros abióticos, os parâmetros bióticos (Árias et al.,2007).

Os Macroinvertebrados Bentônicos (MB) têm sido utilizados, frequentemente, como bioindicadores da qualidade da água. De acordo com a literatura, existem diversas razões para esta utilização: I) possuem hábito sedentário sendo, portanto, representativos da área na qual foram coletados; II) seu ciclo de vida é relativamente curto, refletindo mais rapidamente as alterações do ambiente; III) vivem e se alimentam sobre os sedimentos, área onde as toxinas tendem a se acumular; IV) apresentam alta biodiversidade, o que significa uma maior variabilidade de respostas frente à diferentes tipos de impactos ambientais (Rosenberg \& Resh, 1993; Callisto et al., 2001). Em relação à tolerância frente às adversidades ambientais, são classificados em três grupos principais, existindo exceções dentro de cada grupo, a saber: organismos sensíveis ou intolerantes, organismos tolerantes e organismos resistentes (Goulart \& Callisto, 2003).

Nesta perspectiva, o presente estudo objetivou analisar a influência do uso e ocupação do solo na qualidade da água da sub-bacia do Córrego Machado de Melo, no município de Araçatuba/SP, utilizando macroinvertebrados bentônicos como bioindicadores.

\section{Material e Métodos}

Área de estudo

A identificação do objeto de estudo foi definida utilizando imagens do satélite Landsat 5TM de 26/06/2010, disponibilizadas pelo INPE.

A área escolhida foia sub-bacia do Córrego Machado de Melo, no município de Araçatuba/SP, compreendida entre as coordenadas $50^{\circ} 23^{\prime} 41,34^{\prime \prime} \mathrm{W}, 21^{\circ} 23^{\prime} 12,21^{\prime \prime} \mathrm{S}, 50^{\circ} 28^{\prime} 1,15^{\prime \prime} \mathrm{W}$ e $21^{\circ} 12^{\prime} 30,9^{\prime \prime} \mathrm{S}$.

Os critérios para a escolha das estações amostrais foram: diferenciação no uso e ocupação do solo, representatividade da sub-bacia e facilidade de acesso aos animais.

Para o levantamento das atividades produtivas e de uso e ocupação do solo utilizou-se a técnica de observação sistemática com auxílio do SIG.

\section{Delineamento}

Foram avaliados neste estudo os MB existentes em três estações amostrais, dentre as nascentes que fazem parte da sub-bacia estudada, sendo selecionadas três estações de amostragens (Figura 1). Além dos parâmetros bióticos foram realizadas análises dos parâmetros abióticos: oxigênio dissolvido, turbidez, condutividade, $\mathrm{pH}$, demanda química de oxigênio (DQO), amônio (NH4) e fósforo (P).

A Estação amostral N-187 é uma nascente de um afluente da sub-bacia do Córrego Machado de Melo e representa a região baixa da sub-bacia. $\mathrm{O}$ uso e ocupação do solo foram classificados como LT, porém, esta é a única estação amostral que registrou a presença de vegetação ripária, com predomínio de sedimento arenoso, fluxo médio de correnteza e sem odor.

A Estação amostral N-254 também é uma nascente de um afluente do CórregoMachado de Melo e representa a região média da sub-bacia. O uso e ocupação do solo foram classificados como LT. No entanto, no local de amostragem foram verificados sinais de pastagem para dessedentação de gado. Foi detectado predomínio de sedimento argiloso com fluxo de água bastante lento e com odor forte.

A Nascente 243 é a principal nascente contribuinte do Córrego Machado de Melo, pois representa o alto da sub-bacia. O uso e ocupação do solo foram classificados como $\mathrm{AU}$, sem vegetação ripária próxima ao leito. $\mathrm{O}$ fluxo de água é bastante lento, sem odor e apresenta a proliferação de macrófitas aquáticas.

\section{Procedimentos em campo e laboratório}

As coletas foram realizadas conforme descrito no protocolo para o Biomonitoramento com as Comunidades Bentônicas de Rios e Reservatórios do Estado de São Paulo, fornecido pela CETESB. O procedimento de amostragem ocorreu no sentido inverso ao fluxo do rio, deforma a não interferir nas estações localizadas à montante, ou seja, os pontos mais próximos da nascente foram os últimos a serem coletados. 
Em cada estação amostral foram feitas triplicatas (P1, P2, P3), totalizando nove amostras. A avaliação física e química da água foi realizada pela SANEAR (Saneamento de Araçatuba S.A.) e os parâmetros avaliados foram: oxigênio dissolvido, turbidez, condutividade, $\mathrm{pH}$, demanda química de oxigênio (DQO), amônio (NH4) e fósforo $(\mathrm{P})$.

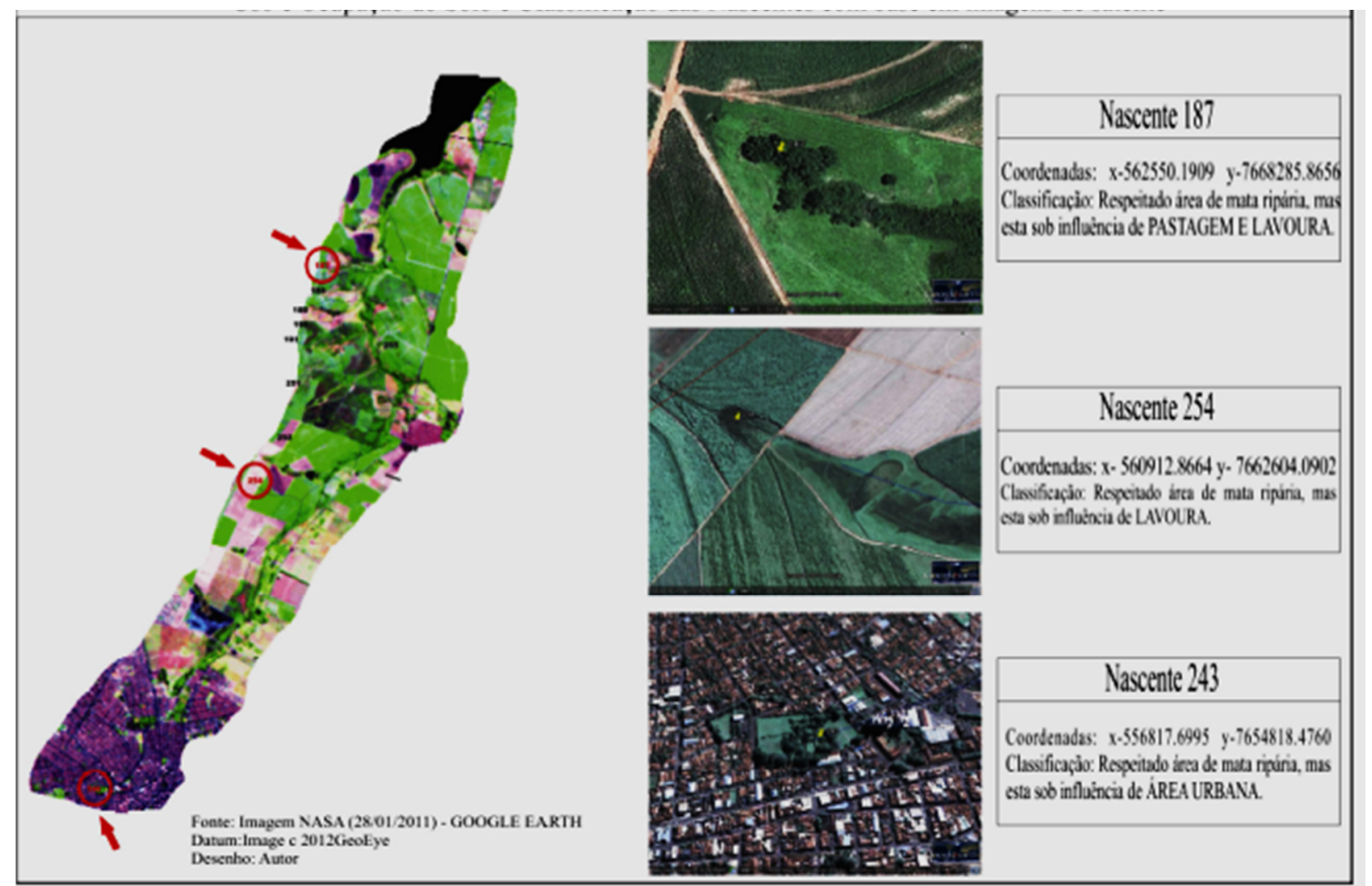

Figura 1. Estações amostrais definidas na sub-bacia do Córrego Machado de Melo, no município de Araçatuba/SP, segundo a classificação do uso e ocupação do solo de pastagem (P), lavoura temporária (LT) e área urbana (AU) (Lima, 2013). Fonte: Arquivo pessoal (2011).

A coleta dos MB foi realizada por meio da Draga de Peterson Modificado, sendo que, ainda em campo, o sedimento coletado foi passado na rede com abertura de malha de $0,2 \mu \mathrm{m}$ para captura dos organismos. Ao término desse procedimento, as amostras foram colocadas dentro de frascos de vidro, sendo adicionada ao material solução de álcool $70 \%$, para conservar os macroinvertebrados, etiquetados com informações dos pontos de coleta para análise posterior em laboratório.

Em laboratório, os sedimentos coletados foram novamente lavados com água corrente em rede de abertura de malha de $0,2 \mu \mathrm{m}$. As folhas e galhos foram subtraídos da amostra lavando-os com cuidado na rede. $\mathrm{O}$ restante da amostra foi transferido para uma bandeja plástica com capacidade para 3 litros, contendo uma solução supersaturada de sal, para promover a flutuação dos organismos, facilitando, assim, sua visualização. O restante do sedimento foi triado sob microscópio estereoscópico para a separação dos macroinvertebrados aquáticos dos detritos vegetais e das partículas inorgânicas dos sedimentos.

Os exemplares triados foram contabilizados, fotografados, conservados em solução de álcool $70 \%$, identificados com auxílio de chaves taxonômicas disponíveis em Costa et al. (2006), e alguns exemplares foram enviados para especialistas da Universidade Estadual de Maringá (UEM) para confirmação da identificação, em nível de família, dos MB (Figura 3).

\section{Resultados}

Foram identificados e distribuídos (em porcentagem) os seguintes usos e ocupações do solo: AU (16,96\%); LT $(42,36 \%)$; P(19,33\%) e F $(16,94 \%)$, conforme a Figura 2. 


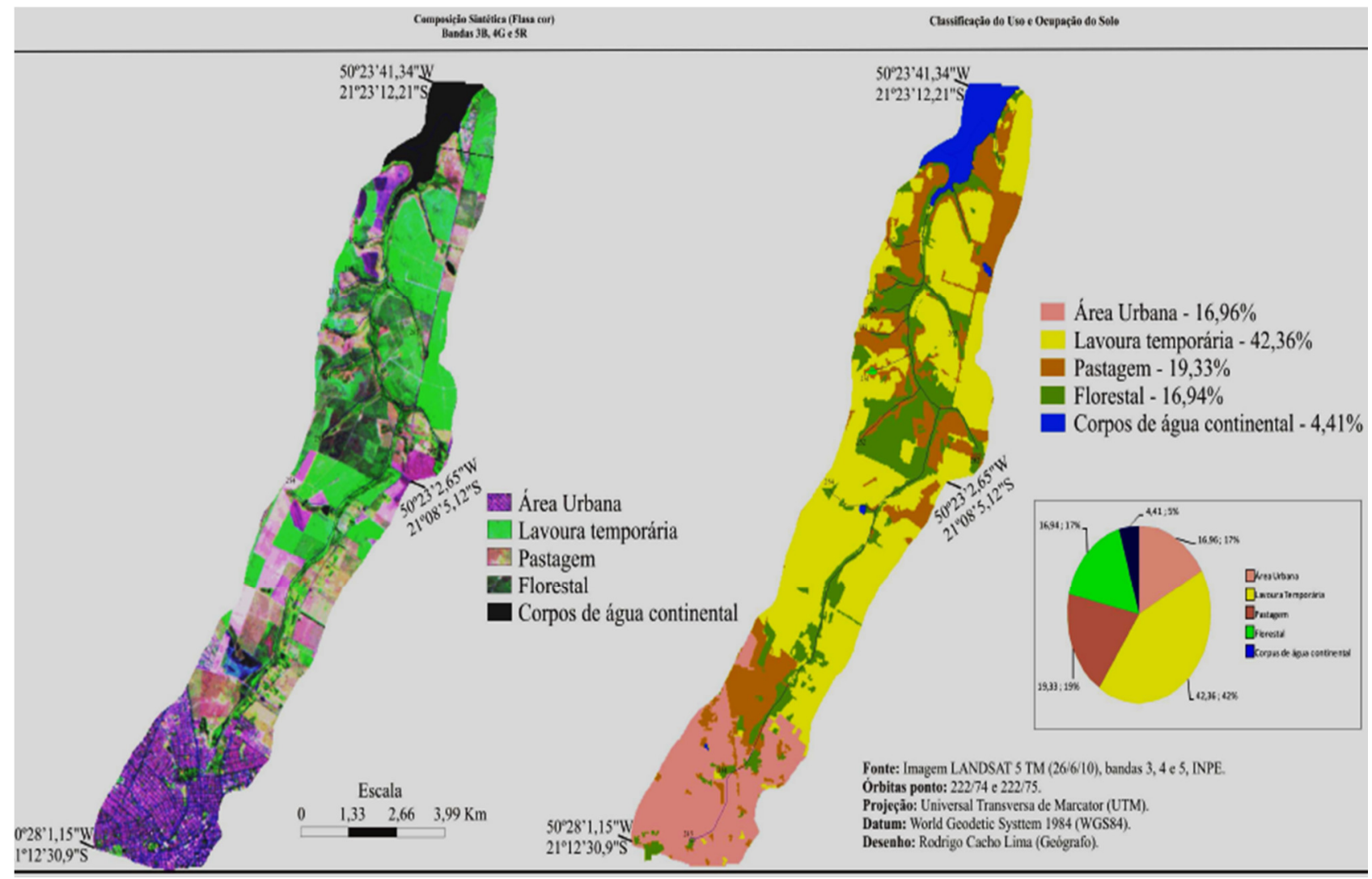

Figura 2. Sub-bacia do Córrego Machado de Melo, no município de Araçatuba/SP, segundo a classificação do uso e ocupação do solo (área total de 57,37 km²) (Lima, 2013). Fonte: Arquivo pessoal (2013).

Os resultados dos parâmetros abióticos foram comparados com os limites estabelecidos pela resolução CONAMA 357/05 (CONAMA, 2005), adotando-se os valores máximos previstos para classe 2 deáguas continentais. As Estações amostrais N-243 e N-254 apresentaram-se alteradas em relação aos parâmetros condutividade elétrica e oxigênio dissolvido, conforme mostra a Tabela 1.

Tabela 1. Parâmetros abióticos mensurados na sub-bacia do Córrego Machado de Melo, no município de Araçatuba/SP, seguindo padrões do CONAMA (2005). Fonte: SANEAR (2013).

\begin{tabular}{|c|c|c|c|c|c|}
\hline Parâmetros & $\begin{array}{c}\text { Padrão } \\
\text { CONAMA }\end{array}$ & Tréplicas & $\begin{array}{l}\text { Estação } \\
\text { N - 187 }\end{array}$ & $\begin{array}{c}\text { Estação } \\
\text { N - 243 }\end{array}$ & $\begin{array}{l}\text { Estação } \\
\text { N - 254 }\end{array}$ \\
\hline \multirow{4}{*}{$\begin{array}{l}\text { Condutividade } \\
\text { elétrica }\left(\mathrm{uS} . \mathrm{cm}^{-1}\right)\end{array}$} & \multirow{4}{*}{$\leq 100$} & Ponto 1 & 55,4 & 202,1 & 116,2 \\
\hline & & Ponto2 & 56,8 & 218,3 & 119,3 \\
\hline & & Ponto 3 & 58,1 & 208,2 & 115,3 \\
\hline & & Média & 56,8 & 116,9 & 209,5 \\
\hline \multirow{4}{*}{$\mathrm{pH}$} & \multirow{4}{*}{$6,0-9,0$} & Ponto 1 & 6,7 & 7,0 & 7,5 \\
\hline & & Ponto2 & 6,7 & 7,1 & 7,6 \\
\hline & & Ponto 3 & 6,9 & 7,0 & 7,5 \\
\hline & & Média & 6,7 & 7,1 & 7,5 \\
\hline \multirow{4}{*}{$\begin{array}{c}\text { Temperatura } \\
\left({ }^{\circ} \mathrm{C}\right)\end{array}$} & \multirow{4}{*}{$4^{\circ} \mathrm{C}-30^{\circ} \mathrm{C}$} & Ponto 1 & 25,1 & 25,5 & 25,1 \\
\hline & & Ponto2 & 25,3 & 25,6 & 25,3 \\
\hline & & Ponto 3 & 25,1 & 25,4 & 25,1 \\
\hline & & Média & 25,2 & 25,5 & 25,2 \\
\hline \multirow{4}{*}{ DQO (mg.L-1 $)$} & \multirow{4}{*}{ - } & Ponto 1 & ND & ND & 30 \\
\hline & & Ponto2 & ND & ND & ND \\
\hline & & Ponto 3 & $\mathrm{ND}$ & $\mathrm{ND}$ & ND \\
\hline & & Média & ND & ND & 10 \\
\hline $\mathrm{NH}_{3}$ & $\geq 5.0$ & Ponto 1 & 1,1 & 1,7 & 6,7 \\
\hline
\end{tabular}


Journal of Environmental Analysis and Progress V. 02 N. 01 (2017) 36-43

\begin{tabular}{|c|c|c|c|c|c|}
\hline \multirow[t]{3}{*}{ (Amônia - mg.L ${ }^{-1}$ ) } & & Ponto2 & 1,1 & 0,9 & 0,6 \\
\hline & & Ponto 3 & 1,0 & 1,1 & 0,5 \\
\hline & & Média & 1,1 & 1,2 & 2,6 \\
\hline \multirow{4}{*}{$\begin{array}{c}\mathrm{O}_{3} \\
\text { (Oxigênio- } \mathrm{mg}^{-L^{-1}} \text { ) }\end{array}$} & \multirow{4}{*}{$\geq 7,0$} & Ponto 1 & 4,8 & 7,1 & 3,2 \\
\hline & & Ponto2 & 5,2 & 7,3 & 2,9 \\
\hline & & Ponto 3 & 5,0 & 7,0 & 3,3 \\
\hline & & Média & 7,1 & 5,0 & 3,1 \\
\hline \multirow{4}{*}{$\begin{array}{c}\mathrm{P} \\
\left(\text { Fósforo- mg.L }{ }^{-1} \text { ) }\right.\end{array}$} & \multirow{4}{*}{$\leq 0,1$} & Ponto 1 & ND & ND & ND \\
\hline & & Ponto2 & ND & ND & ND \\
\hline & & Ponto 3 & ND & ND & ND \\
\hline & & Média & ND & ND & ND \\
\hline
\end{tabular}

A fauna encontrada na sub-bacia do Córrego Machado de Melo, no município de Araçatuba/SP, estava constituída de larvas de
Odonata (subordem Anisoptera) e larvas e adultos de Diptera (família Quironomidae) (Figura 3).
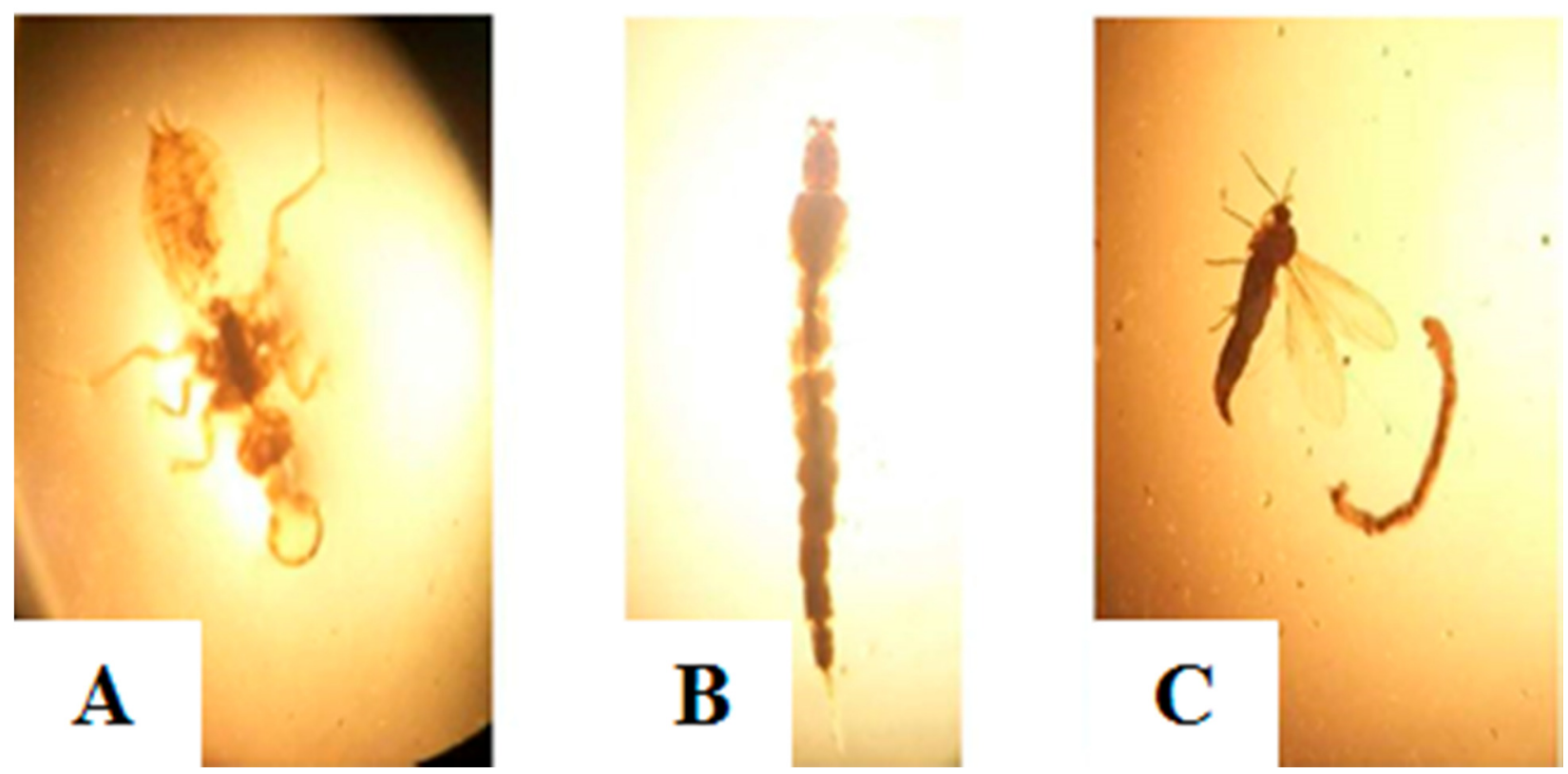

Figura 3. Exemplares de Macroinvertebrados Betônicos (MB) triados nos pontos de coleta da sub-bacia do Córrego Machado de Melo, no município de Araçatuba/SP. A. Larva de Odonata e subordem Anisoptera; B. Larva de Diptera e família Quironomidae; C. Adulto de Diptera ao lado sua larva (Quironomidae). Fonte: Arquivo pessoal (2013).

A fauna de MB registrada nas três estações amostrais foi composta por 263 organismos, sendo $52,1 \%$ dos organismos amostrados na Estação N-187, 44,1\% na Estação $\mathrm{N}-243$; na Estação N-254 foi registrada a ocorrência de apenas 3,8\% dos indivíduos (Tabela 2). A densidade dos organismos de cada amostra foi calculada utilizando-se a fórmula: número de indivíduos/0,0189 $\mathrm{m}^{2}$ (área da draga de Petersen modificado). Na Estação N-187, a família mais representativa foi a Chironomida, com o gênero Stempelina, apresentando uma densidade de 4973,5 ind. $\mathrm{m}^{-2}$. Esta família pertencente ao grupo de indivíduos resistentes (Tabela 3 ).

Tabela 2. Quantidade de Macroinvertebrados Bentônicos (MB) encontrados nas estações amostrais da subbacia do Córrego Machado de Melo, no município de Araçatuba/SP. Fonte: Arquivo pessoal (2013).

\begin{tabular}{|c|c|c|c|c|c|}
\hline Estação Amostral & P1 & $\mathbf{P 2}$ & P3 & Total de Indivíduos & Indivíduos (\%) \\
\hline $\mathrm{N}-187$ & 64 & 54 & 19 & 137 & 52,1 \\
\hline $\mathrm{N}-243$ & 51 & 32 & 33 & 116 & 44,1 \\
\hline $\mathrm{N}-254$ & 7 & 3 & 0 & 10 & 3,8 \\
\hline TOTAL & & & & 263 & 100 \\
\hline
\end{tabular}


Journal of Environmental Analysis and Progress V. 02 N. 01 (2017) 36-43

Tabela 3. Distribuição, número e densidade da fauna bentônica registrada na Estação N-187 da sub-bacia do Córrego Machado de Melo, no município de Araçatuba/SP. Fonte: Arquivo pessoal (2013).

\begin{tabular}{|c|c|c|c|c|c|}
\hline \multirow{2}{*}{ Táxon } & \multicolumn{5}{|c|}{ Quantidade de indivíduos por pontos de coleta } \\
\hline & P1 & $\mathbf{P 2}$ & $\mathbf{P 3}$ & Total & Densidade (ind.m ${ }^{-2}$ ) \\
\hline \multicolumn{6}{|c|}{ Diptera } \\
\hline Chironomidae & 14 & 4 & 14 & 29 & 1534,4 \\
\hline Chironomidae (Stempellina) & 39 & 50 & 5 & 94 & 4973,5 \\
\hline \multicolumn{6}{|c|}{ Molusca } \\
\hline Bivalvia & 0 & 3 & 0 & 3 & 158,7 \\
\hline \multicolumn{6}{|c|}{ Odonata } \\
\hline Anizoptera & 8 & 0 & 0 & 8 & 423,3 \\
\hline \multicolumn{6}{|l|}{ Ephemeroptera } \\
\hline Caenidae & 1 & 0 & 0 & 1 & 52,9 \\
\hline \multicolumn{6}{|c|}{ Trichoptera } \\
\hline Psychomyiidae & 2 & 0 & 0 & 2 & 105,8 \\
\hline TOTAL & & & & 137 & 7248,7 \\
\hline
\end{tabular}

$\mathrm{Na}$ estação N-243, a classe mais representativa foi a Gastrópoda, com uma densidade $3280,4 \mathrm{~m}^{2}$ seguida da família Chironomidae $\left(2116,4 \mathrm{~m}^{2}\right)$. Apesar de esta estação amostral registrar diversidade e densidade equivalente a da estação a N-187, ela se diferenciou pelo fato de que todos os indivíduos amostrados fazem parte de ordens consideradas do grupo resistentes e tolerantes (Díptera, Molusca e Annelida) (Tabela 4).

Tabela 4. Distribuição, número e densidade da fauna bentônica registrada na Estação N-243 da sub-bacia do Córrego Machado de Melo, no município de Araçatuba/SP. Fonte: Arquivo pessoal (2013).

\begin{tabular}{|c|c|c|c|c|c|}
\hline \multirow{2}{*}{ Táxon } & \multicolumn{5}{|c|}{ Quantidade de indivíduos por pontos de coleta } \\
\hline & $\mathbf{P 1}$ & P2 & $\mathbf{P 3}$ & Total & Densidade (ind.m ${ }^{-2}$ ) \\
\hline \multicolumn{6}{|c|}{ Diptera } \\
\hline Chironomidae & 32 & 5 & 3 & 40 & 2116,4 \\
\hline Culicidae (Pupa) & 3 & 2 & 0 & 5 & 264,6 \\
\hline Chironomidae (Stempellina) & 1 & 0 & 0 & 1 & 52,9 \\
\hline \multicolumn{6}{|c|}{ Molusca } \\
\hline Gastropoda & 10 & 24 & 28 & 62 & 3280,4 \\
\hline Bivalvia & 2 & 1 & 1 & 4 & 211,6 \\
\hline \multicolumn{6}{|c|}{ Annelida } \\
\hline Oligochaeta & 2 & 0 & 1 & 3 & 158,7 \\
\hline \multicolumn{6}{|c|}{ Entognatha } \\
\hline Collembola & 1 & 0 & 0 & 1 & 52,9 \\
\hline TOTAL & & & & 116 & 6137,6 \\
\hline
\end{tabular}

$\mathrm{Na}$ Estação N-254, a densidade de organismos foi extremamente baixa, sendo registradas apenas duas classes, Gastrópoda, com densidade de 423,3 ind. $\mathrm{m}^{-2}$ e Oligochaeta com
105,8 ind. $\mathrm{m}^{-2}$, sendo que estes organismos fazem parte do grupo resistente (Tabela 5). 
Tabela 5. Distribuição, número e densidade da fauna bentônica registrada na Estação N-254 da sub-bacia do Córrego Machado de Melo, no município de Araçatuba/SP. Fonte: Arquivo pessoal (2013).

\begin{tabular}{|c|c|c|c|c|c|}
\hline \multirow{2}{*}{ Táxon } & \multicolumn{5}{|c|}{ Quantidade de indivíduos por pontos de coleta } \\
\hline & P1 & $\mathbf{P 2}$ & $\mathbf{P 3}$ & Total & Densidade (ind. $\mathrm{m}^{-2}$ ) \\
\hline \multicolumn{6}{|c|}{ Molusca } \\
\hline Gastropoda & 5 & 3 & 0 & 8 & 423,3 \\
\hline \multicolumn{6}{|c|}{ Annelida } \\
\hline Oligochaeta & 2 & 0 & 0 & 2 & 105,8 \\
\hline TOTAL & & & & 10 & 529,1 \\
\hline
\end{tabular}

\section{Discussão}

De acordo com os resultados encontrados, considerando os parâmetros abióticos, a maioria daqueles avaliados se encontram dentro dos limites estabelecidos pela Resolução do CONAMA $\mathrm{n}^{\mathrm{o}} 357 / 05$ para rios de classe 2 , com exceção da condutividade elétrica e oxigênio dissolvido.

Verificou-se que a Estação amostral N254, classificada com uso e ocupação do solo LT, com influências de $\mathrm{P}$, obteve maior condutividade elétrica, bem acima do limite preconizado. Segundo Nascimento (2012), a condutividade elétrica é a capacidade da água de conduzir corrente elétrica e está relacionada à presença de íons dissolvidos. Registrou-se, ainda, nesta estação, níveis de oxigênio dissolvido (OD) muito abaixo do limite preconizado. $\mathrm{O}$ oxigênio dissolvido é um parâmetro de extrema importância para a análise da poluição de um rio, pois o mesmo é necessário para manter as condições de vida dos seres que vivem na água (Coliischonn \& Tassi, 2008).

Observou-se que dentre as estações amostrais analisadas, a Estação N-254 foi a mais afetada por ações antrópicas, haja vista que seu uso e ocupação do solo se refletem na baixa diversidade de organismos aquáticos e nos valores abióticos registrados. A redução ou aumento da diversidade está relacionada com a saúde do ecossistema e, consequentemente, com o tipo de uso e ocupação do solo (EpaOhio, 1987).

Sugere-se, ainda, a existência de uma forte influência da $\mathrm{AU}$ em ecossistemas aquaticos, uma vez que a vegetação ripária é substituída por loteamentos, como obervado na Estação N-243. Segundo Nascimento (2012), devido a alta tolerância às variações em ambientes impactados, principalmente naqueles de origem urbana, há predominância de organismos do grupo resistente, o que justifica o impacto do uso do solo de AU na qualidade da água. No entando, resultados divergentes foram encontrados no estudo de Santos et al. (2016), realizado em uma área que também possui um contexto de degradação por sua localização na área urbana de Uberaba, na qual apresentou um valor relativamente alto $(\mathrm{n}=$ 10 táxons). Este fato sugere que medidas de densidade podem ser questionadas quanto à sua utilidade (Resh et al., 1995). Magurran (1991) diverge afirmando que há uma diminuição de organismos sensíveis de acordo com o aumento de impactos na água, com uma abundância de organismos tolerantes às condições adversas do ambiente. Neste sentido, pôde-se verificar que as larvas de Chironomidae foram predominantes em todas as estações de amostragens.

Apesar de estar sob influência de LT, a Estação amostral N-187 apresentou-se com vegetação ripária preservada, e os resultados sugerem a importância desta vegetação em função de suas características e dinâmica em ambientes aquáticos. Foi registrada uma maior diversidade e densidade em relação as demais Estações, sendo esta a única estação amostral que registrou a presença de indivíduos do grupo sensível, representados pelas ordens Ephemeroptera e Trichoptera. Este resultado corrobora Goulart \& Callisto (2003), quando relatam que organismos desta ordem possuem uma necessidade de alta concentração de oxigênio na água. Além disso, esses organismos são, normalmente, habitantes de ambientes com heterogeneidade de habitats e são encontrados em águas limpas, sendo considerados como bons indicadores de qualidade da água.

No entanto, fazem-se necessários estudos mais amplos e mais detalhados, através de análises ambientais de grupos de organismos predominantes em outras estações inseridas na sub-bacia do Córrego Machado de Melo, com identificação em nível de espécie e análise estatística.

\section{Conclusão}

Ocorre uma diminuição da diversidade de indivíduos diretamente proporcional ao grau do uso e ocupação do solo, evidenciando o seu impacto na qualidade da água.

A pressão antrópica sobre a biota da subbacia do Córrego Machado de Melo reflete o 
impacto da comunidade de macroinvertebrados bentônicos, principalmente com uma predominância de organismos dos grupos resistentes e/ou tolerantes, considerados indicadores de poluição.

\section{Agradecimentos}

Os autores agradecem à equipe da Empresa de Saneamento de Araçatuba (SANEAR), que viabilizou a realização das análises abióticas, disponibilizando instalações, material e pessoal. Agradeçemos ao Geógrafo Ms. Rodrigo Cacho Lima, pela aplicação da técnica de observação sistemática com auxílio do SIG, permitindo a caracterizaçãodo uso e ocupação do solo da área de estudo.

\section{Referências}

ARIAS, A. R. L.; BUSS, D. F.; ALBURQUERQUE, C.; INÁCIO, A. F.; FREIRE, M. M.; EGLER, M.; MUGNA, R.; BAPTISTA, D. F. 2007. Utilização de bioindicadores na avaliação de impacto e nomonitoramento da contaminação de rios e córregos por agrotóxicos. Ciência \& Saúde Coletiva, v.12, n.1, p.61-72.

CALLISTO, M.; MORETTI, M.; GOULART, M. 2001. Macroinvertebrados Bentônicos como Ferramenta para avaliar a Saúde de Riachos. RBRH - Revista Brasileira de Recursos Hídricos, v.6, n.1, p.71-82.

CETESB, Companhia Ambiental do Estado de São Paulo. 2012. Protocolo para o biomonitoramento com as comunidades bentônicas de rios e reservatórios do estado de São Paulo [recurso eletrônico] / CETESB; Mônica Luisa Kuhlmann ... [et al.]. 113 p. : il. Color.

COLIISCHONN, W.; TASSI, R. 2008. Introdução a Hidrologia. Porto Alegre: UFRG. 151p.

CONAMA. Conselho Nacional do Meio Ambiente. 2005. Resolução $\mathrm{n}^{\circ}$ 357, de 17 de março de 2005. Diário Oficial da UniãoPublicação no DOU n ${ }^{\circ}$ 053, de 18/03/2005. pp.5863.

EPA-OHIO. 1987. Biological criteria for the protection of aquatic life: User's manual for biological field assessment of Ohio surface
Waters. Columbus: Division of water quality monitoring and assessment.

GOUlaRT, M. D. C.; CALlisto, M. 2003. Bioindicadores de qualidade de água como ferramenta em estudos de impacto ambiental. ver. FAPAM, v.2, p.156-164.

NASCIMENTO, F. F. 2012. Riqueza de macroinvertebrados bentônicos e qualidade da água do rio Morozini, no município de Treviso, Santa Catarina. Trabalho de Conclusão de Curso, Universidade do Extremo Sul CatarinenseUNESC. Santa Catarina, Criciúma, Brasil. 45p.

OMETO, J. P. H. B.; MARTINELLI, L. A.; BALLESTER, M. V.; GESSNER, A. F.; KRUSCHE, A. V.; VICTORIA, R. L.; WILLIAMS, M. 2000. Effects of land use on water chemistry and macroinvertebrates in two streams of the Piracicaba river basin, south-east Brazil. Fresh water Biology, v.44, n.2, p.327-337.

QUEIROZ, M. M. F.; IOST, C.; GOMES, S. D.; BOAS, M. A. V. 2010. Influência do uso do solo na qualidade da água de uma microbacia hidrográfica rural. Revista Verde de Agroecologia e Desenvolvimento Sustentável, v.5, n.4, p.200210.

REBOUÇAS, A. C.; BRAGA, B.; TUNDISI, J. G. 2002. Águas doces do Brasil: capital ecológico, uso e conservação. 2 ed. São Paulo, SP. Escrituras Editora. 702p.

RESH, V. H.; NORRIS, R. H.; BARBOUR, M. T.1995. Design and implementation of rapid assessment approaches for water resource monitoring using benthic macroinvertebrates. Australian Journal of Ecology, v.20, p.108-121.

ROSENBERG, D. M.; RESH, V. H. 1993. Introduction to freshwater biomonitoring and benthic macroinvertebrates. In: ROSENBERG, D. M.; RESH, V. H. (Orgs.) Freshwater biomonitoring and benthic macroinvertebrates. New York: Chapman e Hall, pp.1-9.

SANTOS, L. B.; CORREIA, D. L. S.; SANTOS, J. C.2016. Macroinvertebrados bentônicos como bioindicadores do impacto urbano. Journal of Environmental Analysis and Progress, v.1, n.1,p.34-42. 